\title{
INTERPOLATION WITH MEROMORPHIC MATRIX FUNCTIONS
}

\author{
JOSEPH A. BALL AND KEVIN F. CLANCEY
}

(Communicated by Albert Baernstein II)

\begin{abstract}
A complete solution is given to a first-order pole-zero meromorphic matrix function interpolation problem on a closed Riemann surface. The solution to the interpolation problem is constructed from the solution to a natural linear homogeneous system.
\end{abstract}

\section{INTRODUCTION}

In this paper a solution is given to the following first-order pole-zero matrix function interpolation problem on a closed Riemann surface $M$ of genus $g$. Interpolation Problem. Given the data

$$
\mathscr{D}: z_{1}, \ldots, z_{N} ; w_{1}, \ldots, w_{N}: \bar{x}_{1}, \ldots, \bar{x}_{N} ; \underline{y}_{1}, \ldots, \underline{y}_{N}
$$

consisting of $2 N$ distinct points $z_{1}, \ldots, z_{N} ; w_{1}, \ldots, w_{N}$ on $M$, nonzero $r$ dimensional row vectors $\bar{x}_{1}, \ldots, \bar{x}_{N}$, and nonzero $r$-dimensional column vectors $\underline{y}_{1}, \ldots, \underline{y}_{N}$, find necessary and sufficient conditions for there to exist a meromorphic $(r \times r)$-matrix function $G=G(z)$ on $M$ that satisfies:

(1) The only possible poles of any entry of $G$ are simple poles at $w_{1}, \ldots, w_{N}$, and the zero pole divisor of the determinant satisfies (Det $G)=z_{1}+\cdots+z_{N}-$ $w_{1}-\cdots-w_{N}$.

(2) The left kernel $\operatorname{Ker}_{l} G\left(z_{j}\right)$ is the span $\operatorname{Sp}\left(\bar{x}_{j}\right)$ of $\bar{x}_{j}, j=1, \ldots, N$.

(3) The meromorphic matrix function $G^{-1}$ is holomorphic at $w_{j}$, and the right kernel $\operatorname{Ker}\left(G^{-1}\left(w_{j}\right)\right)=\operatorname{Sp}\left(\underline{y}_{j}\right), j=1, \ldots, N$.

In the case of genus zero the scalar version $(r=1)$ of this problem is trivial, and assuming the data points $z_{j}, w_{j}, j=1, \ldots, N$, are not equal to the point at infinity, the matrix problem has a solution if and only if the $N \times N$ matrix

$$
S=\left[\frac{\bar{x}_{i} \underline{y}_{j}}{w_{j}-z_{i}}\right]_{N \times N}
$$

Received by the editors September 18, 1992; this paper was presented at regional meeting \#881 of the American Mathematical Society, Howard University, Washington, D.C., April 17, 1993.

1991 Mathematics Subject Classification. Primary 47A57, 30F10, 14 F05.

Key words and phrases. Interpolation, matrix functions, complex vector bundles.

The work of both authors was supported by grants from the National Science Foundation. 
is invertible. This effective solution of the matrix interpolation problem in the case of genus zero is in [4]. For an extensive treatment of matrix function interpolation problems and their applications on the sphere (see [2]). The solution to the scalar version of the interpolation problem is given by the classical theorem of Abel (see, e.g., [3]). For example, when $M$ has genus 1 , say $M=\mathbf{C} /(\mathbf{Z}+\tau \mathbf{Z})$, $\operatorname{Im} \tau>0$, then the scalar version of the interpolation problem has a solution if and only if $\sum z_{j} \equiv \sum w_{j} \bmod (\mathbf{Z}+\tau \mathbf{Z})$.

Let $D_{o}=p_{1}+\cdots+p_{g}$ be a nonspecial effective divisor of degree $g$ on $M$ consisting of distinct points disjoint from the data points $z_{1}, \ldots, z_{N} ; w_{1}, \ldots, w_{N}$ and $p_{o}$ a fixed point in $M$ disjoint from the data points and the support of $D_{o}$. Note that the generic effective divisor of degree $g$ is nonspecial, and in the case of genus 1 any choice $D_{o}=p_{1}$ is permitted. (See, for example, [3, p. 91].) Let $f_{w_{j}}$ be a nonzero meromorphic function on $M$ with pole-zero divisor satisfying $\left(f_{w_{j}}\right) \geq p_{o}-w_{j}-D_{o}$. Analogous to the matrix $S$ in (1), we introduce

$$
S=\left[\bar{x}_{i} \underline{y}_{j} f_{w_{j}}\left(z_{i}\right)\right]_{N \times N} .
$$

The functions $f_{w_{j}}$ can be considered as automorphic functions on the universal cover $\rho: \tilde{M} \rightarrow M(\tilde{M}$ is taken as the sphere, plane, or unit disc when $g=0$, $g=1$, or $g>1$, respectively), and we let $r_{i j}$ be the residues of $f_{w_{j}}$ at fixed points representing $p_{i}$ in $\tilde{M}, i=1, \ldots, g ; j=1, \ldots, N$. We let $X$ be the $(N \times r)$-matrix with $i$ th row $\bar{x}_{i}, i=1, \ldots, N, Y$ be the $(r \times N)$-matrix with $j$ th column $\underline{y}_{j}, j=1, \ldots, N$ and, for $i=1, \ldots, g, Y_{i}$ be the $(r \times N)$ matrix with $j$ th column $r_{i j} \underline{y}_{j}, j=1, \ldots, N$. The solution to the interpolation problem is contained in the following.

Theorem 1. Necessary and sufficient conditions for the interpolation problem with data $\mathscr{D}$ to have a solution are that the $((N+r g) \times(N+r))$-matrix

$$
T=\left[\begin{array}{cc}
S & X \\
Y_{1} & 0 \\
\vdots & \vdots \\
Y_{g} & 0
\end{array}\right]
$$

have nullity $r$ and the $((N+r g) \times N)$-matrix

$$
S_{g}=\left[\begin{array}{c}
S \\
Y_{1} \\
\vdots \\
Y_{g}
\end{array}\right]
$$

have rank $N$. Moreover, if these condition are satisfied and $\left[\underline{b}_{j}\right], j=1, \ldots, r$, form a basis for the nullspace of $T$, then

$$
G=C+Y \operatorname{Diag}\left(f_{w_{1}}, \ldots, f_{w_{N}}\right) B,
$$

where $B=\left[\underline{b}_{1}, \ldots, \underline{b}_{r}\right]$ and $C=\left[\underline{c}_{1}, \ldots, \underline{c}_{r}\right]$ is the unique solution of the interpolation problem having value $C$ at $p_{o}$.

In the case of genus 1 , when $M=\mathbf{C} /(\mathbf{Z}+\tau \mathbf{Z})$, we can choose

$$
f_{w}(u)=\zeta(u-w)-\zeta\left(u-p_{1}\right)-\left[\zeta\left(p_{o}-w\right)-\zeta\left(p_{o}-p_{1}\right)\right],
$$


where $\zeta=\zeta(u)$ is the Weierstrass $\zeta$-function relative to the lattice $\mathbf{Z}+\tau \mathbf{Z}$. Thus on an elliptic curve we have the following.

Corollary 1. Suppose the data $\mathscr{D}$ is prescribed on the elliptic curve $M=$ $\mathbf{C} /(\mathbf{Z}+\tau \mathbf{Z})$ and $f_{w_{j}}, j=1, \ldots, N$, are given by (6). In order that the interpolation problem with data $\mathscr{D}$ have a solution it is necessary and sufficient that $\operatorname{nullity}(T)=r$ and $\operatorname{rank}\left(S_{1}\right)=N$, where $T$ and $S_{1}$ are the matrices

$$
T=\left[\begin{array}{cc}
S & X \\
Y & 0
\end{array}\right], \quad S_{1}=\left[\begin{array}{l}
S \\
Y
\end{array}\right] .
$$

In case $g \geq 1$, using Abel-Jacobi theory and theta functions it is possible to explicitly describe (after marking $M$ ) the functions $f_{w_{j}}$ and compute their residues $r_{i j}, j=1, \ldots, N, i=1, \ldots, g$. Thus the result in Theorem 1 is both a constructive and computable solution to the interpolation problem. The proof of this theorem given below is elementary. There are interesting connections between the result in this theorem and certain complex vector bundles over $M$. These connections are discussed in the last section.

Proof of Theorem 1. Let us first show the sufficiency of the stated conditions. Suppose $\left[\underline{b}_{j}\right], \quad j=1, \ldots, r$, is a basis for the nullspace of the matrix $T$ appearing in equation (3), and let $G$ be given as in equation (5). Since $Y B=0$, the only possible poles of the entries of $G$ are at the points $w_{1}, \ldots, w_{N}$. The fact that $\left[\begin{array}{l}\underline{b}_{j} \\ \underline{c}_{j}\end{array}\right]$ is in the kernel of the matrix $T$ implies that $\operatorname{Ker}_{l} G\left(z_{j}\right)$ contains $\operatorname{Sp}\left(\bar{x}_{j}\right), j=1, \ldots, N$. Thus every $z_{j}, j=1, \ldots, N$, is a zero of Det $G$. In local coordinates $z=z(p)$ with $z\left(w_{j}\right)=0$ near $w_{j}$,

$$
G(z)=\frac{\underline{y}_{j} \bar{v}}{z}+\text { [analytic], }
$$

where $\bar{v}$ is an $r$-dimensional row vector, $j=1, \ldots, N$. It follows that $\operatorname{Det} G$ has at most simple poles at $w_{1}, \ldots, w_{N}$. Since the degree of Det $G$ is zero, we conclude that the divisor of $\operatorname{Det} G$ is $(\operatorname{Det} G)=z_{1}+\cdots+z_{N}-w_{1}-\cdots-w_{N}$. In particular, $G$ satisfies condition (1) of the interpolation problem. Moreover, the vector $\bar{v}$ appearing in (7) must be nonzero, and $G$ satisfies condition (3) of the interpolation problem. Finally, since each $z_{j}, j=1, \ldots, N$, is a simple zero of Det $G$, condition (2) of the interpolation problem holds. This concludes the proof of the sufficiency.

In order to see the necessity of the stated conditions, observe that any solution of the interpolation problem necessarily has the form (5). This is again a consequence of the fact that near $w_{j}, j=1, \ldots, N$, any solution $G$ of the interpolation problem has the local form (7) with $\bar{v}$ nonzero. Thus $G$ can be expressed in the form (5), where the residue conditions at $p_{j}, j=1, \ldots, g$, force $Y B=0$ and condition (2) for interpolation forces

$$
T\left[\begin{array}{l}
B \\
C
\end{array}\right]=0 .
$$

Since $C=G\left(p_{o}\right)$ is invertible, it follows that the kernel of $T$ is at least of dimension $r$. If the matrix $S_{g}$ does not have rank $N$, then there is a second matrix $\tilde{B}$ such that

$$
T\left[\begin{array}{l}
\tilde{B} \\
C
\end{array}\right]=0,
$$


and using the sufficiency of the stated conditions the meromorphic matrix function

$$
\tilde{G}=C+Y \operatorname{Diag}\left(f_{w_{1}}, \ldots, f_{w_{N}}\right) \tilde{B}
$$

would be a second solution to the interpolation problem with $G\left(p_{o}\right)=C$. This contradicts the obvious uniqueness. Thus $S_{g}$ must have rank $N$, and the kernel of $T$ is of dimension $r$. The proof of the theorem is complete.

\section{EXAMPLES}

We give some applications of Theorem 1 in the case of genus 1 .

Example 1. Consider on $M=\mathbf{C} /(\mathbf{Z}+\tau \mathbf{Z})$ two-dimensional data

$$
\mathscr{D}: \quad z_{1}, z_{2} ; w_{1}, w_{2}: \bar{x}_{1}, \bar{x}_{2} ; \underline{y}_{1}, \underline{y}_{2} .
$$

The interpolation problem with data $\mathscr{D}$ has a solution if and only if $z_{1}+z_{2} \equiv$ $w_{1}+w_{2} \bmod (\mathbf{Z}+\tau \mathbf{Z}), \bar{x}_{1}=c \bar{x}_{2}, \underline{y}_{1}=d \underline{y}_{2}$, for some nonzero $c, d$, and $\bar{x}_{1} \underline{y}_{1} \neq 0$.

Example 2. Suppose $g=1, N \leq r$, and $\bar{x}_{1}, \ldots, \bar{x}_{N}\left(\right.$ or $\left.\underline{y}_{1}, \ldots, \underline{y}_{N}\right)$ are linearly independent. Then the interpolation problem with data $\mathscr{D}$ does not have a solution.

Example 3. Again suppose $M=\mathbf{C} /(\mathbf{Z}+\tau \mathbf{Z})$. Suppose the data is scalar. Abel's Theorem says there is a solution of the interpolation problem with zeros $z_{1}, \ldots, z_{N}$ and poles $w_{1}, \ldots, w_{N}$ if and only if $\sum z_{i} \equiv \sum w_{i} \bmod (\mathbf{Z}+\tau \mathbf{Z})$. Theorem 1 establishes that a solution to this scalar interpolation problem exists if and only if the $((N+1) \times(N+1))$-matrix

$$
T=\left[\begin{array}{cccc}
f_{w_{1}}\left(z_{1}\right) & \cdots & f_{w_{N}}\left(z_{1}\right) & 1 \\
\vdots & & \vdots & \vdots \\
f_{w_{1}}\left(z_{N}\right) & \cdots & f_{w_{N}}\left(z_{N}\right) & 1 \\
1 & \cdots & 1 & 0
\end{array}\right]
$$

has a one-dimensional nullspace and the first $N$ columns of $T$ are linearly independent. Moreover, given a solution $\left[\frac{b}{c}\right]$ of $T \underline{x}=\underline{0}$ the function

$$
f=c+\sum_{j=1}^{N} b_{j} f_{w_{j}}
$$

has divisor $(f)=z_{1}+\cdots+z_{N}-w_{1}-\cdots-w_{N}$. A similar matrix version of Abel's Theorem can be formulated for any genus.

We note that the conditions in Theorem 1 that the nullity of $T$ is $r$ and $S_{g}$ has rank $N$ are equivalent to the condition that the null space of $T$ has the form

$$
\left\{\left[\begin{array}{c}
R \underline{c} \\
\underline{c}
\end{array}\right]: \underline{c} \in \mathbf{C}^{r}\right\}
$$

for some $(N \times r)$-matrix $R$. Thus the solutions of the interpolation problem have the form

$$
G=C+Y \operatorname{Diag}\left(f_{w_{1}}, \ldots, f_{w_{N}}\right) R C
$$


where $C$ varies over $\operatorname{GL}(r, C)$. Since the degree of Det $G$ is zero for any solution of the interpolation problem, we must conclude the rows of the matrix $R$ appearing above are all nonzero.

\section{CONNECTIONS WITH COMPLEX VECTOR BUNDLES}

It is natural to associate with the data $\mathscr{D}$ a Weil divisor $\theta_{\mathscr{D}}$ and corresponding rank $r$ complex vector bundle $E_{\mathscr{D}}$ over $M$. The Weil divisor $\theta_{\mathscr{D}}$ at $p$ in $M$ is the germ of the regular meromorphic $(r \times r)$-matrix function $L^{-1}$, where $L$ denotes a local solution to the interpolation problem at $p$. The concept of Weil divisor used here is consistent with the definition in [5]. The (linear equivalence class of the) divisor $\theta_{\mathscr{D}}$ corresponds to (a holomorphic equivalence class of ) a vector bundle $E_{\mathscr{D}}$ over $M$. If $\left\{L_{\alpha}\right\}_{\alpha \in A}$ is a family of local solutions of the interpolation problem relative to an open cover $\left\{V_{\alpha}\right\}_{\alpha \in A}$ of $M$, then $\Phi_{\alpha \beta}=L_{\alpha}^{-1} L_{\beta}$ on $V_{\alpha} \cap V_{\beta}$ define coordinate transformations for the vector bundle $E_{\mathscr{D}}$.

It is easily seen that a solution to the interpolation problem exists if and only if the associated vector bundle $E_{\mathscr{D}}$ is trivial. Further, a proof of Theorem 1 can be based on this fact and the result in Lemma 1 given below.

The description of the holomorphic sections of $E_{\mathscr{D}}$ is the following.

Lemma 1. Holomorphic sections of $E_{\mathscr{D}}$ are in one-to-one correspondence with $r$-dimensional meromorphic vector functions on $M$ having the form

$$
\underline{h}=\underline{c}+\sum_{j=1}^{N} b_{j} \underline{y}_{j} f_{w_{j}},
$$

where the $(N+r)$-dimensional column vector $\left[\frac{b}{\underline{b}}\right]$ is in the nullspace of the matrix $T$ given in (3).

Proof. Let $\left\{L_{\alpha}\right\}_{\alpha \in A}$ denote a family of local solutions to the interpolation problem relative to some open cover $\left\{V_{\alpha}\right\}_{\alpha \in A}$ of $M$ and $\Phi_{\alpha \beta}=L_{\alpha}^{-1} L_{\beta}$ coordinate transformations for the bundle $E_{\mathscr{D}}$. There is a natural isomorphism between holomorphic sections $\left\{\underline{t}_{\alpha}\right\}_{\alpha \in A}$ of $E_{\mathscr{D}}$ and global $r$-dimensional vector meromorphic functions $\underline{h}$ on $M$ satisfying $\underline{t}_{\alpha}=L_{\alpha}^{-1} \underline{h}$ is holomorphic on $V_{\alpha}$, $\alpha \in A$. The fact that the right-kernel of $L_{\alpha}^{-1}\left(w_{j}\right)=\operatorname{Sp}\left(\underline{y}_{j}\right), j=1, \ldots, N$, allows one easily to conclude that any such $\underline{h}$ has the form (8). In local coordinates $z$ near $z_{i}$ with $z\left(z_{i}\right)=0$, the local interpolating matrix function $L_{\alpha}$ has the form

$$
L_{\alpha}^{-1}(z)=\frac{\underline{u} \bar{x}_{i}}{z}+\{\text { holomorphic }\}
$$

where $\underline{u}$ is a nonzero column vector. Thus the requirement of analyticity of $L_{\alpha}^{-1} \underline{h}$ at $z_{i}, i=1, \ldots, N$, forces the vector $\left[\frac{b}{c}\right]$ to be in the kernel of the $(N \times(N+r))$-matrix $[S X]$. Residue considerations at $p_{1}, \ldots, p_{g}$ imply $Y_{i} \underline{b}=\underline{0}, i=1, \ldots, g$. Conversely, if $\underline{h}$ has the form (8) with $\left[\frac{b}{c}\right]$ in the kernel of $T$, then $\underline{t}_{\alpha}=L_{\alpha}^{-1} \underline{h}, \alpha \in A$, defines a holomorphic section of $E_{\mathscr{D}}$.

One nice consequnce of the above lemma is the formula

$$
\operatorname{Dim} \Gamma\left(E_{\mathscr{D}}\right)=\operatorname{Dim} \operatorname{Ker}(T)
$$


which gives the dimension of the space $\Gamma\left(E_{\mathscr{D}}\right)$ of holomorphic sections of $E_{\mathscr{D}}$ in terms of the nullity of the matrix $T$.

It should be noted that very little except the convenient formalism of vector bundles is being used here. On the other hand, in genus 1 using the classical works of Weil [5] and Atiyah [1], the authors have made connections between interpolation data such as $\mathscr{D}$ and the "building block" bundles $F_{r}$ of Atiyah [1]. These connections will be described elsewhere.

\section{REFERENCES}

1. M. F. Atiyah, Vector bundles over an elliptic curve, Proc. London Math. Soc. 7 (1957), 414-452.

2. J. A. Ball, I. Gohberg, and L. Rodman, Interpolation of rational matrix functions, Birkhäuser-Verlag, Basel, Berlin, and Boston, 1990.

3. H. M. Farkas and I. Kra, Riemann surfaces, Springer-Verlag, New York, 1980.

4. I. Gohberg, M. A. Kaashoek, L. Lerer and L. Rodman, Minimal divisors of rational matrix functions with prescribed zero and pole structure, Topics in Operator Theory Systems and Networks, Birkhäuser-Verlag, Basel, Berlin, and Boston, 1984, pp. 241-275.

5. A. Weil, Généralization des fonctions abéliennes, J. Math. Pures Appl. 17 (1938), 47-87.

Department of Mathematics, Virginia Tech, Blacksburg, Virginia 24601

E-mail address: ball@math.vt.edu

Department of Mathematics, University of Georgia, Athens, Georgia 30602

E-mail address: kclancey@joe.math.uga 